\title{
Membrane imaging by simultaneous second-harmonic generation and two-photon microscopy: errata
}

\author{
L. Moreaux \\ Laboratoire de Physiologie, Ecole Supérieure de Physique et Chimie Industrielles, Institut National de la Santé et de la Recherche \\ Médicale, Equipe Postulante 00-02, 10 Rue Vauquelin, 75005 Paris, France

\section{O. Sandre} \\ Laboratoire Physico-Chimie Curie, Centre National de la Recherche Scientifique, Unité Mixte de Recherche 168, \\ 11 Rue Pierre et Marie Curie, 75005 Paris, France
}

\section{Blanchard-Desce}

Département de Chimie, Ecole Normale Supérieure, Centre National de la Recherche Scientifique, Unité Mixte de Recherche 8640, 24 Rue Lhomond, 75005 Paris, France

\section{J. Mertz}

Laboratoire de Physiologie, Ecole Supérieure de Physique et Chimie Industrielles, Institut National de la Santé et de la Recherche Médicale, Equipe Postulante 00-02, 10 Rue Vauquelin, 75005 Paris, France

In Ref. 1, Eq. (2) should read as

$$
\Theta=\frac{3}{8 \pi} \int S(\theta, \varphi) \mathrm{d} \Omega \approx \frac{3 \xi^{2}}{4 k_{\omega}^{2} w_{x} w_{z} \sqrt{1-\xi^{2}}},
$$

Eq. (3) should read as

$$
\sigma_{\mathrm{SHG}}=\frac{4 \hbar \omega^{5}}{3 \pi n \epsilon_{0}{ }^{3} c^{5}} \beta^{2} \quad\left[\mathrm{~m}^{4} / \text { photon } \mathrm{s}^{-1}\right],
$$

and the right-hand side of Eq. (4) should be multiplied by $2 \sqrt{2}$.

Finally, our discussion on the N.A. dependence of $P_{\mathrm{SHG}}$ is somewhat erroneous. In fact, $P_{\mathrm{SHG}}$ is roughly independent of N.A. for low N.A.'s.

\section{Reference}

1. L. Moreaux, O. Sandre, M. Blanchard-Desce, and J. Mertz, Opt. Lett. 25, 320 (2000). 